\title{
Illusory Paschen curves associated with strongly electronegative gases
}

\author{
McAllister, lain Wilson
}

Published in:

IEEE Transactions on Electrical Insulation

Link to article, DOI:

10.1109/14.85109

Publication date:

1991

Document Version

Publisher's PDF, also known as Version of record

Link back to DTU Orbit

Citation (APA):

McAllister, I. W. (1991). Illusory Paschen curves associated with strongly electronegative gases. IEEE

Transactions on Electrical Insulation, 26(3), 391-397. https://doi.org/10.1109/14.85109

\section{General rights}

Copyright and moral rights for the publications made accessible in the public portal are retained by the authors and/or other copyright owners and it is a condition of accessing publications that users recognise and abide by the legal requirements associated with these rights.

- Users may download and print one copy of any publication from the public portal for the purpose of private study or research.

- You may not further distribute the material or use it for any profit-making activity or commercial gain

- You may freely distribute the URL identifying the publication in the public portal

If you believe that this document breaches copyright please contact us providing details, and we will remove access to the work immediately and investigate your claim 


\title{
Illusory Paschen Curves Associated with Strongly Electronegative Gases
}

\author{
I. W. McAllister \\ Physics Laboratory II, \\ The Technical University of Denmark, Lyngby, \\ Denmark
}

\begin{abstract}
For a strongly electronegative gas, breakdown voltage measurements made on a moderately non-uniform field test gap give rise to an apparently linear $\left(U_{s}, p d\right)$ curve. The curve can be designated a Paschen curve, but the erroneous nature of this designation becomes apparent from a linear regression analysis of the experimental breakdown data. An explanation for this anomaly is brought forth through the application of an analytical linear-regression analysis to the theoretical breakdownvoltage relationship. This latter analysis indicates that, for moderately non-uniform field test gaps, the gradient of the theoretical breakdown-voltage curve is given approximately by $\eta(E / p)_{l i m}$, where $\eta$ is the field utilization factor of the test gap. $(E / p)_{l i m}$ is the pressure-reduced limiting electric field strength for the gas in question and the value of the Paschen-curve gradient.
\end{abstract}

\section{INTRODUCTION}

$\mathrm{O}$ NE of the principal uses of a test gap is in the determination of the Paschen curve. Although Paschen's law refers to breakdown in a uniform field, all practical test gaps produce, to a greater or lesser degree, a non-uniform field. Despite this inherent limitation, Karlsson and Pedersen [1] have shown that valid Paschen curve data can still be obtained, provided the initial electron avalanche growth traverses the entire gap. If this condition is upheld, the Paschen curve for a strongly electronegative gas is found to be linear over the $p d$ range of interest to practical situations, see [2-5]. $p$ is the gas pressure and $d$ is the test gap spacing. In such circumstances, the test gap has been classified as producing a weakly non-uniform field [6].

In many studies, the test gap adopted provides only a moderately non-uniform field such that, although direct breakdown still occurs, the initial electron avalanche growth does not traverse the entire gap [6]. Despite this discharge restriction, an apparently linear breakdown voltage curve can be obtained [7] which is then mistakenly identified as a Paschen curve. It is only by extracting the relevant parameter values from the experimental data that the anomalous nature of such a curve becomes apparent [8]. In the present paper the underlying features of such illusory Paschen curves are sought.

\section{DISCHARGE ONSET VOLTAGE}

Gor a non-uniform field gap, the discharge onset volt$\mathrm{F}$ age $U_{0}$ of a strongly electronegative gas may be expressed as the product of the field utilization factor $\eta$, the surface roughness factor $\xi$, the surface curvature factor $\zeta$, the gas pressure $p$, the gap length $d$ and the $(E / p)_{\text {lim }}[9]$; viz.

$$
U_{0}=\eta \xi \zeta\left(\frac{E}{p}\right)_{l i m p} p d
$$


Depending on the degree of electric field non-uniformity, the gas in question and its pressure, $U_{0}$ will represent either the corona onset voltage or the minimum breakdown voltage. For a moderately nonuniform field, it is the latter, i.e. $U_{s} \equiv U_{0}$.

The field utilization factor [10] is a function of the macroscopic gap geometry alone and is defined as

$$
\eta \equiv \frac{U}{E_{a} d}
$$

with $E_{a}$ being the maximum field strength of the idealized macroscopic geometry associated with a potential difference $U$. The normalized parameters $\xi$ and $\zeta$ are related to both the electrode geometry and the gas in question. However, as test gaps with carefully prepared electrode surfaces are under discussion, the effects of electrode surface roughness upon $U_{s}$ will not be considered further, and hence in this study the value of $\xi$ will be taken as unity. Thus it is only $\zeta$ which requires to be considered in detail.

\subsection{SURFACE CURVATURE FACTOR $\zeta$}

This parameter expresses the fact that, for a non-uniform field, the discharge onset field strength $E_{0}$ for ideal electrodes is greater than the limiting value $E_{l i m}$ of the gas in question [11]. This behavior is accounted for by introducing a dimensionless parameter, namely the surface curvature factor $\zeta$, which is defined by

$$
\frac{E_{0}}{p} \equiv \zeta\left(\frac{E}{p}\right)_{l i m}
$$

with $\zeta \geqslant 1$. This factor is so named because, under conditions of practical interest, the fulfillment of the streamer criterion [12] is, with respect to the gap geometry, a function of the mean curvature $H$ of the electrode surface at the location of $E_{a}$ alone. Thus $E_{0}$ is rendered independent of both the remainder of this electrode geometry and the overall geometry of the gap. It should be noted that $E_{0}$ is the value of $E_{a}$ at discharge onset. The above situation arises under the following circumstances.

If $E(s)$ represents the field strength along the field line associated with $E_{a}$ and $s$ is a distance coordinate along this field line measured from the electrode surface $(s=0)$, then

$$
E(s)=E_{a} f(H s)
$$

where $f(H s)$ represents the spatial variation of $E(s)$ with reference to the normalized distance $H s$. For a regular surface, the mean curvature $H$ is defined as

$$
2 H \equiv \frac{1}{R_{1}}+\frac{1}{R_{2}}
$$

where $R_{1}$ and $R_{2}$ are the principal radii of curvature at the surface location of interest. The complete variation of $E(s)$ is of course controlled by both the gap geometry and the applied potentials. However, for $H s<0.1$, the Green differential equation [13] relating the electric field and the differential geometry of equipotential surfaces ensures that $E(s)$ is effectively dependent on $H$ alone, and thus $E(s)$ can be approximated by

$$
E(s)=E_{a}(1-2 H s)
$$

This situation implies that when the initial electron avalanche growth is confined to the proximity of the highly stressed electrode, then $E_{0}$ will be a function of $H$ alone, all other aspects being equal.

With reference to strongly electronegative gases, it is found that upon application of the streamer criterion to the above field distribution one obtains

$$
\zeta \approx 1+\sqrt{\frac{4 M}{p / H}}
$$

and

$$
H s_{0} \approx \sqrt{\frac{M}{p / H}}
$$

see $[11,14]$ for details. The parameter $M$ represents the figure of merit for a strongly electronegative gas [12], while $H s_{0}$ is the normalized critical avalanche length. This latter parameter, which represents the integration path length associated with avalanche growth, satisfies the following relationship

$$
\zeta f\left(H s_{0}\right)=1
$$

It should be noted that, because the derivation of (7) is based on the assumption that $H s_{0}<0.1,(8)$ implies that $M /(p / H)<0.01$.

\section{ANALYTICAL REGRESSION ANALYSIS}

Gor moderately non-uniform fields, the variation of the Iheoretical $U_{s}$ with $p d$ is a shallow curve, see Figure 1 . (Note that for clarity, the degree of curvature has been greatly exaggerated). In practice, owing to the unavoidable scatter which normally occurs with experimental $U_{s}$ values, it will be very difficult to identify such a curved $U_{s}$ variation. The more natural reaction would be to undertake a linear regression analysis of the experimental $U_{s}$ data; i.e. to treat the data as though these constituted a valid set of Paschen curve measurements. On the basis of the theoretical $U_{s}$ variation, the parameters of a linear regression fit will now be determined. 


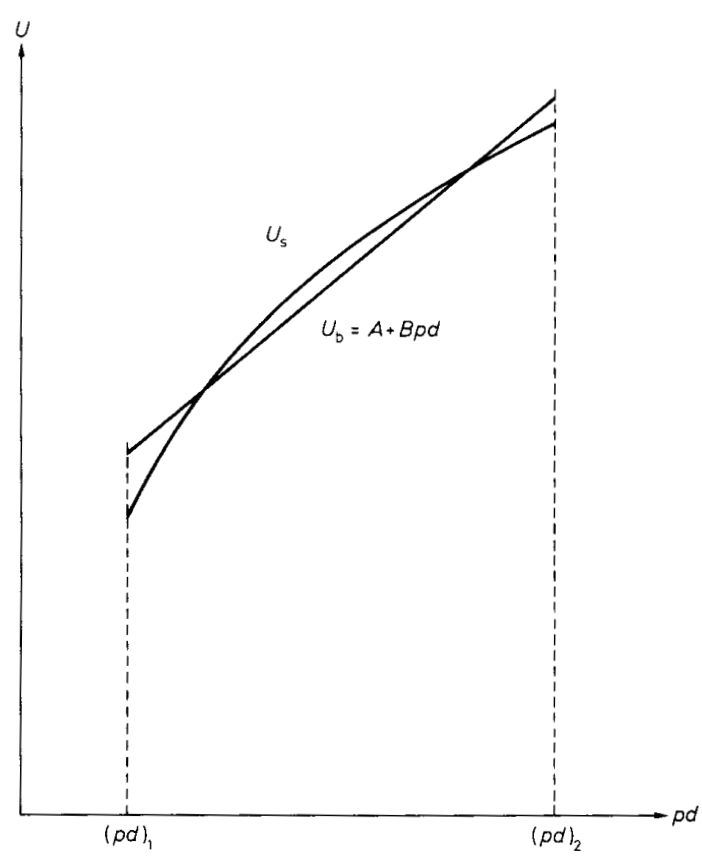

Figure 1. Basis of regression analysis.

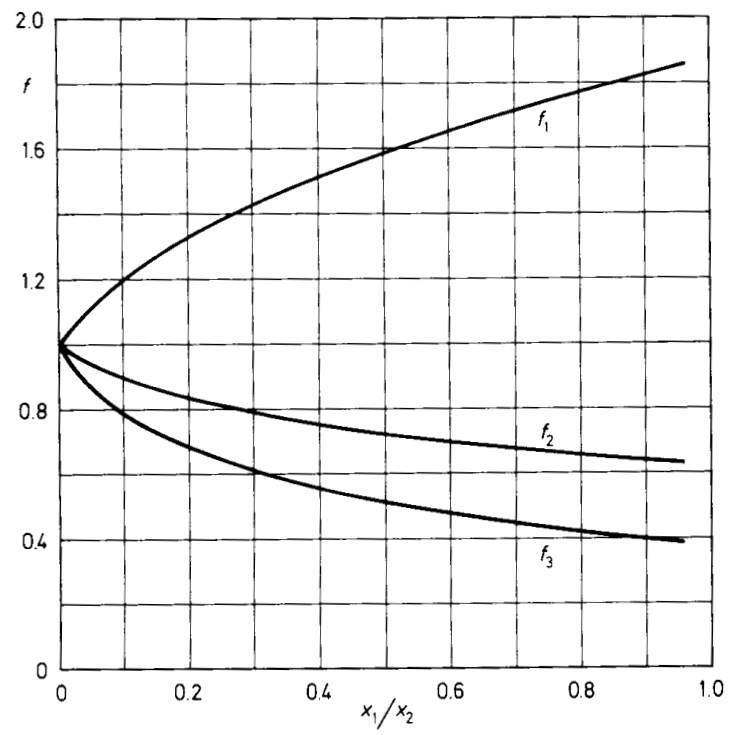

Figure 2.

Variation of $f_{1}, f_{2}$ and $f_{3}$ with the ratio $x_{1} / x_{2}$.

We apply the analysis to the theoretical breakdown voltage relationship $U$, which is a function of the independent variable $p d$, and an approximate breakdown voltage relationship $U_{b}$. As we are concerned with a linear regres- sion analysis, $U_{b}$ is assumed to be a linear function of $p d$, see Figure 1. In the present discussion, it is convenient to replace $p d$ with the variable $x$, and hence we have

$$
U_{s}=f(x)
$$

and

$$
U_{b}=A+B x
$$

To determine $A$ and $B$ we employ the method of least squares.

If the deviation is defined as the difference between the true value and the predicted value, then, with respect to discrete numerical data, the method is concerned with minimizing the sum of the squared deviations [15]. For a continuous function, we can express the equivalent sum as an integral and thus, for a finite range of $x$ values, this can be expressed as

$$
F=\int_{x_{1}}^{x_{2}}[f(x)-(A+B x)]^{2} d x
$$

where $x_{2}$ and $x_{1}$ are the upper and lower values of the $x$ range of interest. The function $F$ can be minimized by deriving $\partial F / \partial A$ and $\partial F / \partial B$, and equating these partial derivatives to zero. Thereafter the solution of the two simultaneous equations provides $A$ and $B$, viz.

$$
\begin{gathered}
A=\frac{2}{\left(x_{2}-x_{1}\right)^{4}}\left[2\left(x_{2}^{3}-x_{1}^{3}\right) \int_{x_{1}}^{x_{2}} f(x) d x\right. \\
\left.-3\left(x_{2}^{2}-x_{1}^{2}\right) \int_{x_{1}}^{x_{2}} x f(x) d x\right] \\
B=\frac{6}{\left(x_{2}-x_{1}\right)^{3}}\left[2 \int_{x_{1}}^{x_{2}} x f(x) d x\right. \\
\left.-\left(x_{2}+x_{1}\right) \int_{x_{1}}^{x_{2}} f(x) d x\right]
\end{gathered}
$$

By analogy with numerical analysis, the coefficient of determination $r^{2}$ can be employed as a measure of how accurately the linear relationship fits the function $f(x)$. This parameter is defined by the ratio [15]

$$
r^{2}=\frac{N}{N+F}
$$

where $N$, which is often called the explained variation, is given by

$$
N=\int_{x_{1}}^{x_{2}}(A+B x-G)^{2} d x
$$


The parameter $G$ is the mean value of $f(x)$, i.e.

$$
G=\frac{1}{\left(x_{2}-x_{1}\right)} \int_{x_{1}}^{x_{2}} f(x) d x
$$

and in relation to $N, F$ is sometimes referred to as the unexplained variation.

A general expression for $r^{2}$ can be obtained by substituting the relevant expressions for $A, B$ and $G$ into (15). This leads to

$$
r^{2}=\frac{B^{2}\left(x_{2}-x_{1}\right)^{3}}{12\left[\int_{x_{1}}^{x_{2}} f^{2}(x) d x-G^{2}\left(x_{2}-x_{1}\right)\right]}
$$

For the gap geometries of interest we assume that, as a first approach, $U_{s}$ can be obtained from (1) with (7). Hence if we set $S=\eta(E / p)_{l i m}$ and $C=S \sqrt{4 M H d}$, then, with respect to the present analysis, we have

$$
f(x)=S x+C \sqrt{x}
$$

It should be emphasized that the selection of $S$ and $C$ as constants implies that each of their constituent parameters, i.e. $\eta, H d, M$ and $(E / p)_{l i m}$ is also constant.

The non-fulfillment of this condition would imply that $p d$ could not be used as the controlled variable in the regression analysis. The choice of another controlled variable can, however, lead to a new dependent variable and thus a different $f(x)$.

Upon substitution of (19) into (13), (14) and (18) and evaluating the integrals, we obtain

$$
\begin{gathered}
A=\frac{4}{15} f_{1} C \sqrt{x_{2}} \\
B=S\left[1+\frac{4}{5} f_{2} D\right]
\end{gathered}
$$

and

$$
r^{2}=\frac{\left[1+(4 / 5) f_{2} D\right]^{2}}{1+(8 / 5) f_{2} D+(2 / 3) f_{3} D^{2}}
$$

where, for $\mu=x_{1} / x_{2}$ and $\mu<1$,

$$
\begin{gathered}
f_{1}=\frac{1+\mu-10 \mu^{3 / 2}+10 \mu^{2}-\mu^{5 / 2}-\mu^{7 / 2}}{(1-\mu)^{3}} \\
f_{2}=\frac{1-5 \mu+5 \mu^{3 / 2}-\mu^{5 / 2}}{(1-\mu)^{3}}
\end{gathered}
$$

and

$$
f_{3}=\frac{1-9 \mu+16 \mu^{3 / 2}-9 \mu^{2}+\mu^{3}}{(1-\mu)^{4}}
$$

and

$$
D=\frac{C}{S \sqrt{x_{2}}}
$$

To gain an appreciation of the behavior of $f_{1}, f_{2}$ and $f_{3}$, the variation of each function with $\left(x_{1} / x_{2}\right)$ is shown in
Figure 2. In practice, the $p d$ range studied is generally more than a decade and thus $\left(x_{1} / x_{2}\right)<0.1$. From Figure 2 this implies that the values of $f_{1}, f_{2}$ and $f_{3}$ are $\approx 1$.

Upon substitution for $S$ and $C$ we obtain the expressions relevant to the present discussion, viz.

$$
\begin{aligned}
& A=\frac{4}{15} f_{1} D \eta(E / p)_{l i m} p_{2} d \\
& B=\eta(E / p)_{l i m}\left[1+\frac{4}{5} f_{2} D\right]
\end{aligned}
$$

with $D=\sqrt{4 M /\left(p_{2} / H\right)}$. This $D$ is used in (22) to determine $r^{2}$. In deriving (27) and (28), we assumed that, as frequently occurs in practice, $d$ is held constant and thus $x_{2}=p_{2} d$.

Table 1

Analytically and numerically derived values of $A$ $[\mathrm{kV}], B\left[\mathrm{kV}(\mathrm{mm} \mathrm{MPa})^{-1}\right]$, and $r^{2}$.

\begin{tabular}{|r|cc|cc|}
\hline \hline & \multicolumn{2}{|c|}{$p_{1} / p_{2}=0.125$} & \multicolumn{2}{c|}{$p_{z_{1}} / p_{z_{2}}=0.120$} \\
\hline & analytical & numerical & analytical & numerical \\
\hline$A$ & 2.96 & 4.13 & 2.99 & 5.50 \\
$B$ & 66.2 & 69.3 & 65.6 & 65.9 \\
$r^{2}$ & 0.99996 & 0.9997 & 0.99996 & 0.9994 \\
\hline
\end{tabular}

Table 2.

Measured $U$, values [kV] of Berger and Senouci [7] and the calculated normalized critical avalanche lengths $s_{0} / d[8]$ and $s_{0} / R$ at pressure $p[\mathrm{MPa}$ ].

\begin{tabular}{|c|c|c|c|}
\hline \hline$p$ & $U_{s}[7]$ & $s_{0} / d[8]$ & $s_{0} / R$ \\
\hline 0.025 & 11.5 & 0.243 & 0.152 \\
0.050 & 21 & 0.168 & 0.105 \\
0.075 & 29 & 0.136 & 0.085 \\
0.10 & 40 & 0.117 & 0.073 \\
0.15 & 56.5 & 0.095 & 0.059 \\
0.20 & 73.5 & 0.082 & 0.051 \\
0.25 & 91 & 0.073 & 0.046 \\
0.30 & 108 & 0.067 & 0.042 \\
0.35 & 126 & 0.062 & 0.039 \\
0.40 & 142 & 0.058 & 0.036 \\
\hline \hline
\end{tabular}

\subsection{APPLICATION OF THE ANALYSIS}

To illustrate the present analysis, we will refer to the experimental study undertaken by Berger and Senouci [7], in which a point/plane gap was employed to investigate Paschen's law for $\mathrm{SF}_{6}$. The severe limitations associated 
with this choice of test gap for Paschen curve measurements has been fully discussed by McAllister and Crichton [8]. Thus although the $U_{s}$ measurements of Berger and Senouci cannot be associated with Paschen's law, the measurements are, with respect to the present analysis, valid data.

In [7], Berger and Senouci employed a hemisphericallyended rod of tip radius $R=8 \mathrm{~mm}$ at a constant distance $d=5 \mathrm{~mm}$ from a plane electrode such that $\eta=3.56 / 5=$ 0.712 . For such a point electrode, $H=1 / R$. The pressure range investigated was 0.025 to $0.4 \mathrm{MPa}$. For $\mathrm{SF}_{6}$, we have $(E / p)_{l i m}=88.6 \mathrm{kV}(\mathrm{mm} \mathrm{MPa})^{-1}$ and $M=0.004$ $\mathrm{mm} \mathrm{MPa} \mathrm{[16],} \mathrm{and} \mathrm{hence} \mathrm{the} \mathrm{condition} M / p R \leqslant 0.01$ is only upheld for $p \geqslant 0.05 \mathrm{MPa}$. Thus for the pressure range 0.05 to $0.4 \mathrm{MPa}$, we obtain using (27), (28) and (22) the values for $A, B$ and $r^{2}$ listed in Table 1, column 1 .

Berger and Senouci tabulate in their paper [7] the corresponding measured $U_{s}$ values and these are listed in Table 2. On applying a numerical linear regression analysis to these data for the same pressure range, we obtain the values given in Table 1, column 2 .

From the first and second columns of Table 1, it is seen that the agreement between the $B$ values is much better than between the $A$ values. This situation is a direct consequence of the fact that the experimental $A$.value is very dependent on the absolute accuracy of the voltage measurement, while the corresponding $B$ value is only dependent on the relative accuracy of this measurement. Although no information about these measurement accuracies is given by Berger and Senouci [7], the relative accuracy is generally much better than the absolute accuracy. In relation to the $A$ values of Table 1 it should be noted that $A$ (Paschen) is only $0.35 \mathrm{kV}$ [16].

Although $\mathrm{SF}_{6}$ is a non-ideal gas, with respect to the breakdown analysis, this feature may be taken into account by replacing $p$ with the compressibility-corrected gas pressure $p_{z}$, where $p_{z}$, is given by

$$
p_{z}=\frac{p}{Z(p, T)}
$$

$Z(p, T)$ is the compressibility factor $(\leqslant 1)$ for the gas at pressure $p$ and temperature $T$. Values for this factor can be derived from the expressions given by Vibholm and Mollerup [17]; see also the Appendix in [18]. In turn the $p_{z}$ substitution leads for $\mathrm{SF}_{6}$ to $\left(E / p_{z}\right)_{l i m}=87.9 \mathrm{kV}(\mathrm{mm}$ $\mathrm{MPa})^{-1}$ at $20^{\circ} \mathrm{C}[18]$.

Upon re-evaluation of the 'analytical' coefficients together with a re-analysis of the measured data, the coefficient values shown in Table 1 , columns 3 and 4 were obtained. By taking into account the nonideal behavior of $\mathrm{SF}_{6}$, the agreement between the new $B$ values improves, while for the $A$ value agreement deteriorates. With the analytical values the small change $(-1 \%)$ in the $B$ value reflects an equally small change (+1\%) in the $A$ values (columns 1 and 3 ). For the numerical data, a reduction of $5 \%$ in $B$ is associated with an increase of $33 \%$ in the $A$ values (columns 2 and 4 ). This behavior is a consequence of the fact that the regression fits to the experimental data $\left(r^{2}=0.999\right)$ are not as good as those obtained with the analytical fit $\left(r^{2}=0.9999\right)$. The values of $r^{2}$ obtained with the analytical regression analysis are such that, for practical purposes, the breakdown voltage curve for a moderately non-uniform field test gap is linear.

\section{DISCUSSION}

$\mathrm{C}^{\mathrm{Rom}}$ the outset, it must be stated that the linear breakdown voltage curves under discussion are not Paschen curves, although owing to their high degree of linearity they can be mistaken for such. On the basis of this linearity, an analytical linear regression analysis was applied to the theoretical breakdown voltage relationship for moderately non-uniform fields. This enabled expressions for the linear regression coefficients to be derived. These coefficients represent the slope and constant term of the equivalent linear relationship. Coefficients evaluated with these expressions are shown to provide values which are in agreement with the values derived from a numerical analysis of experimental data.

The slope of the linear curve is given by (28). For $(4 / 5) f_{2} D \ll 1$, it is evident that this slope closely approximates to $\eta(E / p)_{l i m}$. This value can also be deduced directly via the $\zeta$ expression. As $M /(p / H)$ tends to 0 for increasing values of $p, \zeta \rightarrow 1$ and thus from (7) and (1) we have $U_{s} \rightarrow \eta(E / p)_{\text {lim }} p d$, i.e. the $\left(U_{s}, p d\right)$ curve tends asymptotically to a straight line for which the gradient is $\eta(E / p)_{l i m}$.

From Table 2, it is evident that, for the Berger and Senouci gap, where electron avalanche growth occurs over a small fraction of the gap only, $s_{0} / d<0.25$, the concept of $\zeta$ can be successfully employed.

If however, the avalanche growth occurs over a significant fraction of the gap, e.g. $s_{1} / d>0.5,(6)$ will no longer be a valid approximation for $E(s)$ and hence $E_{0}$ will not be a function of $H$ alone. In such conditions, it is necessary to derive a new relationship for the ratio $E_{0} / E_{l i m}$. 
As with the $\zeta$ expression, (7), $E_{0} / E_{l i m}$ will be expressible in the form

$$
\frac{E_{0}}{E_{\text {lim }}}=1+P(p, M, H, d)
$$

where $P$ represents a perturbation term. Consequently, with reference to the present analysis, $(30)$ implies that only the second term of (19) requires alteration prior to the derivation of the corresponding expressions for $A, B$ and $r^{2}$. Nevertheless the gradient of the breakdown voltage curve will remain approximately $\eta(E / p)_{l i m}$.

To assess any set of published data, the preceding analysis indicates that, apart from the general experimental data, a knowledge of $\eta$ for the test gap employed is also required. This value is seldom quoted directly. In the following, we will discuss an approach which allows a reliable estimate of $\eta$ to be obtained, without recourse to involved numerical computations.

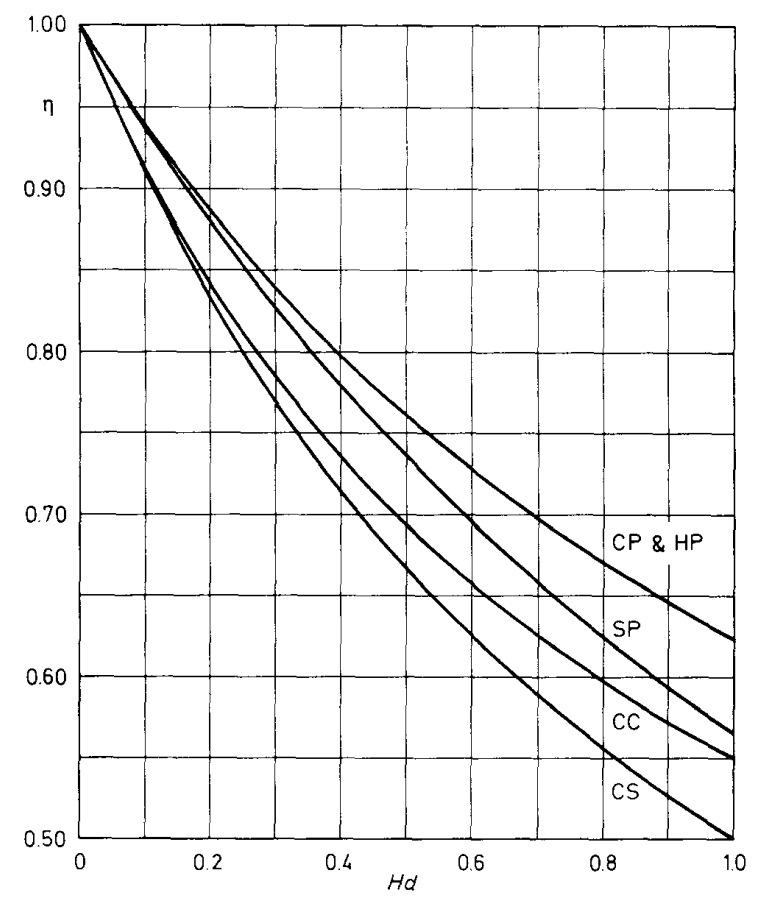

Figure 3.

Variation of field utilization factor $\eta$ for moderately non-uniform field gaps. CS: concentric sphere gap, CC: concentric cylinder gap, SP: sphere/plane gap, CP: cylinder/plane gap, HP: hyperboloid point/plane gap.

\subsection{FIELD UTILIZATION FACTOR ASSOCIATED WITH MODERATELY NON-UNIFORM FIELDS}

Whether a particular gap can be classified as moderately non-uniform is dependent not only on its geometric features, but also upon the gas in question and the gas pressure. For the present discussion, the classification will be taken to apply to the range $0.1<H d \leqslant 1$. A reliable estimate of the $\eta$ value can be obtained from a study of the variation of $\eta$ with $H d$ for several relevant gap geometries, viz. the concentric sphere gap, the concentric circular-cylinder gap, the isolated sphere/plane gap, and the isolated circular-cylinder/plane gap.

Although the above gap geometries may appear rather impractical, these gaps encompass the range of geometric features necessary for the provision of a monotonic field distribution. The associated $\eta$ distributions for the aforementioned geometries are illustrated in Figure 3.

To produce a non-uniform field, one of the electrodes should possess a finite (non-zero) $H$. The simplest electrode geometries which exhibit the overall $\left(R_{1}, R_{2}\right)$ variations of interest are the sphere: $R_{1}$ finite, $R_{2}$ finite and $R_{1}=R_{2} ;$ and the circular cylinder: $R_{1}$ finite, $R_{2}$ infinite

To ensure a monotonic field distribution, the other electrode must either be of a finite $H$ of the same sign, or have $H=0$, i.e. a plane. From Figure 3, it is evident that the concentric gap geometries provide the more non-uniform field distributions, and that cylindrical electrodes are associated with less non-uniform fields than with those of spherical geometry.

The majority of test gaps are of the sphere/plane type, e.g. see [19]. Hence owing to the existence of supporting shafts, the $\eta$ values of interest will lie between the two HV electrode/plane curves shown in Figure 3, as does the $\eta$ value from Berger and Senouci [7]. As an assessment of these $\eta$ limits, the variation of $\eta$ for a hyperboloid point/plane gap was investigated, i.e. an electrode with an infinitely long supporting shaft. From this study, it was discovered that, with respect to $H d$, the $\eta$ variation for this gap was identical with that for a cylinder/plane gap. Hence the $\eta$ variations depicted in Figure 3 must represent the limits for all practical (monotonic) test gaps.

Thus, from a knowledge of the electrode geometry and gap spacing alone, $\eta$ can be estimated reliably without recourse to an actual field solution. Moreover, in the absence of sufficient field data, this $\eta$ approach allows a critical evaluation of published breakdown data to be 
undertaken. The Berger and Senouci [7] pressure- and $\eta$-corrected experimental data for $\mathrm{SF}_{6}$ yield an $\left(E / p_{z}\right)_{l i m}$ value approximately $7 \%$ higher than the Paschen value of $87.9 \mathrm{kV}(\mathrm{mm} \mathrm{MPa})^{-1}$. If the perturbation term in the theory were taken into account, this discrepancy would be reduced.

\section{CONCLUSION}

TSING an analytical regression analysis, it has been shown that, for a strongly electronegative gas, the (direct) breakdown voltage curve obtained with a moderately non-uniform field is effectively linear. The slope of such curves however are shown to be $\left\langle(E / p)_{\text {lim }}\right.$. For this reason, breakdown voltage curves which exhibit these features have been designated illusory Paschen curves.

Based on the regression analysis presented, it is demonstrated that a reliable estimate of the Paschen curve gradient of $(E / p)_{\text {lim }}$ can be made from moderately nonuniform field breakdown voltage data, through a knowledge of the $\eta$ value limits associated with the $H d$ value of the relevant test gap.

\section{REFERENCES}

[1] P. W. Karlsson and A. Pedersen, "Inherent Limitations in Uniform Field Discharge Data for $\mathrm{SF}_{6}$ ", IEEE Trans. Power Appar. \& Syst., Vol. 91, pp. 1597-1601, 1972.

[2] J. Berril, J. M. Christensen and I. W. McAllister, "Measurement of the Figure of Merit $M$ for Several Perfluorocarbon Gases", in L. G. Christophorou and D. W. Bouldin (eds.), Gaseous Dielectrics V, Pergamon Press New York, pp. 304-310, 1987.

[3] J. Berril, J. M. Christensen and I. W. McAllister, "Measurement of the Figure of Merit $M$ for $\mathrm{CBrClF}_{2}, \mathrm{CBr}_{2} \mathrm{~F}_{2}, \mathrm{CBrF}_{3}$ and $\mathrm{CClF}_{3}$ ", Fifth International Symposium on HV Engineering, Braunschweig 1987, Paper 15.16.

[4] J. Berril and I. W. McAllister, "Measurement of the Figure of Merit $M$ for Several $\mathrm{N}_{2}$-Based Binary Gas Mixtures", Sixth International Symposium on HV Engineering, New Orleans 1989, Paper 32.08.

[5] J. Berril and I. W. McAllister, "Measurement of the Figure of Merit $M$ for Several $\mathrm{SF}_{6}$-Based Binary Gas Mixtures", in L. G. Christophorou and I. Sauers (eds.), Gaseous Dielectrics VI, Plenum Publishing New York, pp. 193-199, 1991.

[6] I. W. McAllister, "On the Choice of a Test Gap for Strongly Electronegative Gases and Gas Mixtures", in L. G. Christophorou and M. O. Pace (eds.), Gaseous Dielectrics IV, Pergamon Press New York, pp. 195-202, 1984.
[7] G. Berger and B. Senouci, "The Role of Impurities on the Deviation from Paschen's Law of $\mathrm{SF}_{6}$ ", J. Phys. D: Appl. Phys., Vol. 19, pp. 2337-2342, 1986.

[8] I. W. McAllister and G. C. Crichton, "The Concept of Paschen's Law with Reference to $\mathrm{SF}_{6}$ ", J. Phys. D: Appl. Phys., Vol. 20, pp. 1537-1539, 1987.

[9] W. Mosch and W. Hauschild, "Möglichkeiten zur Berechnung der Durchschlagspannung schwach inhomogener Anordnungen im $\mathrm{SF}_{6}$ ", Elektrie, Vol. 28, pp. 152-156, 1974.

[10] A. Schwaiger, "Beitrag zur elektrischen Festigkeitslehre", Archiv für Elektrotechnik, Vol. 11, pp. 4150, 1922.

[11] A. Pedersen and E. Bregnsbo, "Estimation of Breakdown Voltages in Compressed $\mathrm{SF}_{6}$ ", Internationales Symposium Hochspannungstechnik, Zurich 1975, pp. $432-436$.

[12] A. Pedersen, I. W. McAllister, G. C. Crichton and S. Vibholm, "Formulation of the Streamer Breakdown Criterion and its Application to Strongly Electronegative Gases and Gas Mixtures", Archiv für Elektrotechnik, Vol. 67, pp. 395-402, 1984.

[13] I. W. McAllister and A. Pedersen, "Green's Differential Equation and Electrostatic Fields", J. Phys. D: Appl. Phys., Vol. 21, pp. 1823-1825, 1988 and Vol. 22, p. 473, 1989.

[14] I. W. McAllister, "The Influence of Electrode Macroscopic Curvature upon Surface Roughness Effects in Compressed $\mathrm{SF}_{6}$ ", Archiv für Elektrotechnik, Vol. 62, pp. 43-49, 1980.

[15] C. Chatfield, Statistics for Technology, Penguin Books Harmondsworth 1970.

[16] J. Berril, J. M. Christensen and A. Pedersen, "Measurement of the Figure of Merit Related to the Effect of Electrode Surface Defects on Breakdown for Strongly Electronegative Gases or Gas Mixtures", Seventh International Conference on Gas Discharges and their Applications, London 1982, pp. 266-268.

[17] S. Vibholm and J. Mollerup, "On the Compressibility Factor for $\mathrm{SF}_{6}$ ", Sixth International Conference on Gas Discharges and their Applications, Edinburgh 1980, IEE Conference Publication No. 189, part 1, pp. 278-280, 1980.

[18] I. W. McAllister and G. C. Crichton, "An Experimental Method to Determine the Electrostatic Field Enhancement Factor of a Practical Conductor Surface", IEEE Trans. Elect. Insul., Vol. 24, pp. 325-333 and p. 572, 1989.

[19] ASTM Standard D 2477, Standard Test Method for Dielectric Breakdown Voltage and Dielectric Strength of Insulating Gases at Commercial Power Frequencies, ASTM Philadelphia 1979. 